\title{
DESCRIPCIÓN CUANTITATIVA Y CUALITATIVA DEL ESPECTADOR DE VÍDEOS EN YOUTUBE ESPAÑA
}

\author{
Quantitative and qualitative description of video viewers on Youtube Spain
}

\author{
Autor: GALLARDO CAMACHO, Jorge \\ Profesor de la Facultad de Comunicación de la Universidad Camilo José Cela \\ jgallardo@ucjc.edu / gallardo.jorge@gmail.com
}

\section{Resumen}

Esta investigación analiza el perfil del espectador de vídeos en Internet desde un prisma cuantitativo pero también cualitativo. El artículo se centra en el caso de Youtube España y recopila las investigaciones del autor sobre la materia para aportar una descripción del usuario de la que es la mayor red social de vídeos. La vertiente cualitativa permitirá conocer datos relevantes además del sexo o la edad de los usuarios. Por ejemplo, la investigación concluye, entre otros factores, que el usuario de Youtube importa a la red los mismos gustos y formas de consumo que adopta ante las industrias culturales tradicionales. Retratamos con datos empíricos el perfil del usuario de Youtube España y abrimos nuevas líneas de investigación.

\section{Palabras clave}

Youtube España; Internet; video; audiovisual; redes sociales; audiencias; industrias culturales

\begin{abstract}
This research analyzes the profile of videos viewers on the Internet from a quantitative point of view but also qualitative. The article focuses on the case of Youtube Spain and collects the author's researches about the subject to provide a full description of the user in the largest social network of video. The qualitative aspect will reveal aspects more important than sex or age of users. For example, the research concludes, among other factors, that the Youtube user imports the same tastes and consumption patterns that adopts from the traditional cultural industries. Empirical data reflect the Youtube user profile to open new lines of research in the Communication Area.
\end{abstract}

\section{Key words}

Youtube Spain; Internet; video; audiovisual; social networks; audiences, cultural industries

\section{Introducción}

Mientras la descripción de los espectadores de la televisión tradicional ha sido analizada desde un gran número de estudios e investigaciones, bien es cierto que existe una grave carencia en el análisis de los internautas que ven vídeos. Es paradójico que esto sea así ya que la televisión tradicional se limita a una muestra de paneles de audimetría con 4.625 hogares en España (Kantar Media, 2013), algo que contrasta con la potencialidad de captación de datos más certeros en la red (cuantificación real de visitas, tiempo de conexión en la web, etc.). En este artículo vamos a analizar los estudios tanto cuantitativos como cualitativos más destacados para describir el perfil del usuario de Youtube España. Pero iremos más allá de su edad o de su nivel sociodemográfico. El problema ante el que nos enfrentamos es que la información que se maneja y los resultados que se difunden por parte de las consultorías y empresas del sector giran en torno solo al ámbito cuantitativo. Pero, epor qué Youtube España es nuestro objeto de estudio? Nos centraremos en quién y cómo consume Youtube España. La red social de vídeos crece exponencialmente 
cada mes: con 4.000 millones de vídeos vistos cada día (el doble que en 2010), 60 nuevas horas de vídeo subidos por minuto (un $37 \%$ más en tan solo seis meses de 2012) y 400 millones de visitas al día desde teléfonos y tabletas (Youtube, 2012). Las visitas procedentes de España en Youtube suponen el 2,5\% del total (Alexa, 2013). Youtube asegura en su web que "800 millones de personas de todo el mundo ven su red social cada mes". Sin embargo, Youtube aún no da beneficios.

Para afrontar este problema, Youtube ofrece a sus anunciantes grandes audiencias pero también nichos y grupos definidos entre sus consumidores (los clasifica en grupos: amantes de la música, aficionados a la comedia, a los videojuegos o a los coches). Los contenidos de Youtube "nos proporcionan una amplia radiografía sobre los gustos y tendencias de consumo de la red en general" (Lavado, 2010: 89). La red intenta conocer a sus usuarios para ofrecer una publicidad personalizada para cada anunciante. En esa búsqueda de la rentabilidad se hace necesaria la capacidad de describir quién ve los cientos de miles de vídeos que almacena. Y esa información es importante que se difunda entre los investigadores de las Ciencias de la Comunicación. Y es que el uso de redes sociales es el segundo servicio más utilizado por los internautas $(60,3 \%)$ después del correo electrónico (88\%), según el Estudio General de Medios (AIMC, 2012a). El sitio más visitado es, con diferencia, la red social de vídeos Youtube con 15.887.000 millones de visitas en un mes. Seis de cada diez internautas españoles encuestados por el Interactive Advertising Bureau (IAB, 2010) visita con regularidad Youtube. Esta red social de vídeos solo es superada por Facebook (el 89\% la visita con asiduidad). Pero en este artículo nos vamos a centrar en un perfil más amplio del espectador de vídeos en la red social Youtube.

\subsection{El espectador y el consumo audiovisual de lo glocal en Youtube}

Existen investigaciones que aseguran que Internet pondrá en peligro la identidad cultural de las regiones. Que lo global arrasará el valor de lo local. Es el caso de Fernández (2002) cuando asegura que la concentración empresarial hacia la que nos impulsa la globalización puede aplastar nuestra cultura más cercana. O’Reilly (2006) se suma a esta concepción de la eliminación de lo singular cuando asevera que Internet se convierte con las webs 2.0. (como Youtube) en un cerebro colectivo. Internet se caracteriza por ser el medio en el que convergen el resto de medios y que tiene el mayor rasgo de globalidad. Pero, ¿el espectador de vídeos de Internet da la espalda a lo local ante esta potencialidad de consumo global?

Hay teóricos que no ven la globalidad de la red como un enemigo de la identidad cultural. Mattelart (1993: 31) asegura que "aunque la universalización reestructura lo cercano, lo cierto es que se mantiene lo singular como elemento clave del proceso". En este sentido, Mattelart apoya el retorno a lo singular frente a la globalización. Pero hay más teóricos que han dado al contenido audiovisual, antes de su expansión en Internet, la capacidad de reflejar el significado y el significante de una realidad cultural específica. Es decir, que lo audiovisual puede reflejar una cultura acotada y, en definitiva, más local (Geertz, 1988). Y eso, trasladado a Internet, puede suceder.

Los grupos sociales tienen la oportunidad de crear sus aldeas locales, pero también tienen la posibilidad de mantener su estatus en la aldea global.

\subsection{El espectador de contenidos televisivos en Internet}

Para conocer más el perfil del espectador es importante saber qué tipo de contenidos consume, es decir, hay que ir más allá de la procedencia física del vídeo. En esta batalla entre la espontaneidad de Internet y la influencia de las industrias culturales tradicionales, ¿quién gana? Las cadenas de televisión ponen en marcha mecanismos para trasladar a Internet su influencia y poder en el ámbito audiovisual. Existe un "pulso de poder por dominar la industria cultural que parece que va a converger en Internet" (Gallardo, 2010: 185). Burgess y Green (2009) van más allá cuando aseguran que Youtube es el nuevo campo de lucha de poder del siglo XXI. Añaden que está siendo manipulado no solo por las industrias de medios de comunicación sino también por el público, por grupos de presión y por productores audiovisuales independientes.

De hecho, las cadenas de televisión han sobrepasado la fase denominada por Mariano Cebrián (2004: 207) como de "aproximación a Internet". Bustamante 
(2006: 263) observó un peligro para la televisión cuando planteó que la fragmentación de la oferta implicaba una degradación de la base económica para la fabricación de programas de calidad. Algo que podría "acabar con los canales generalistas, una experiencia compartida que hoy sustenta el consenso y la cohesión social". Pero vamos a ver que se produce una traslación de los gustos del espectador de vídeos a Internet y que se mantiene esa "experiencia compartida" de la que habla Bustamante.

Horkheimer y Adorno (1994) advierten de que "la industria cultural es la industria de la diversión” y, por tanto, los espectadores van a recurrir a los mismos prescriptores de contenidos audiovisuales: las cadenas de televisión. Nos encontramos ante lo que Javier Pérez de Silva (2000: 51) denomina la tercera revolución industrial en la que "la convergencia TV+PC, aunque evidente, no permite augurar un cambio de actitud radical por parte de los telespectadores".

\subsection{La actitud pasiva del espectador de vídeos ante Internet}

A continuación pasamos a repasar los estudios que analizan el comportamiento del espectador de vídeos en Internet. Con esta parte se intenta romper con una idea preconcebida: los espectadores de Internet interactúan siempre. En este sentido, Pérez y Santos (2009) aseguran que, en estas webs, el usuario abandona su rol pasivo porque se le permite establecer formas de diálogo más fluidas con los medios y pasa a ser un generador de contenidos dentro del proceso de comunicación. Sin embargo, otros autores como Díaz (2009) advierten de que el vídeo apenas comienza a desarrollar un lenguaje propio en el ciberespacio e invita a la comunidad investigadora a resolver la relación entre el usuario y los vídeos con preguntas como: "¿puede el vídeo desarrollar un nuevo lenguaje audiovisual en el ciberespacio al servicio de la información liberadora?" (Díaz, 2009: 71). Otros investigadores (Ritzer, 1996; Robison y Geofrey, 1997; Wolton, 2000; Owen, 2000) vaticinan un rol pasivo del internauta como espectador de contenidos audiovisuales en la red.

Youtube (2012) asegura en su web que "cada semana 100 millones de personas realizan una acción social en Youtube" (utilizar la función "Me gusta", "compartir vídeos" o "realizar comentarios"). Además la red social de vídeos añade que más de la mitad de los vídeos de Youtube se han puntuado o incluyen comentarios de la comunidad, y que cada día millones de vídeos se marcan como "favoritos". Sin embargo, tras estos datos globales, se esconde una baja interactividad por parte de los usuarios.

Bill Tancer (2008: 125) comprobó que solo el 0,16\% de las personas que visitaron Youtube subieron vídeos. Pero nosotros nos vamos a centrar en modos de interacción más sencillos (que requieren un conocimiento informático inferior): como valorar o comentar un vídeo.

\section{Objetivos de la investigación}

Ante este breve marco teórico introductorio, esta investigación tiene un único objetivo, claro y conciso:

- O1. Elaborar una descripción cuantitativa y cualitativa del espectador de vídeos de Internet en el sitio web Youtube España.

Para obtener este objetivo principal será necesario recopilar la información cuantitativa existente para describir al usuario. Pero, con respecto al perfil cualitativo del usuario, también plantearemos otros tres objetivos secundarios. Es decir, vemos importante sumar a la descripción del usuario de Youtube España tres perspectivas vinculadas a esos tres objetivos secundarios:

- O1.1. Demostrar el interés del espectador por su identidad cultural, por lo cercano y por el concepto de lo glocal a pesar de la globalidad de Youtube.

- O1.2. Demostrar el interés del espectador por contenidos elaborados por las industrias culturales tradicionales. ¿Se decanta más por los contenidos procedentes de la televisión tradicional que por los generados por los usuarios? 
- O1.3. Demostrar y analizar la actitud pasiva del espectador de vídeos en Internet. ¿Renuncia el usuario a la potencialidad de interacción de la red?

La metodología se centra en la recopilación y análisis de las investigaciones realizadas hasta el momento sobre el perfil del usuario de Youtube. Con esta investigación intentamos ir hacia lo cualitativo a través de lo cuantitativo. Recurrimos a una metodología cuantitativa tras la observación del objeto de estudio en investigaciones científicas publicadas con un contexto en común: Youtube España. Por tanto, este artículo supone la confluencia de todas las investigaciones realizadas por el autor en la Universidad San Jorge de Zaragoza, en la Universidad Camilo José Cela y en el grupo de investigación "Comunicación y Poder” de la Universidad de Málaga. Es, precisamente, el análisis global de esta producción científica el que facilitará la obtención de un perfil del espectador de vídeos en Internet.

La metodología cuantitativa relacionada con los objetivos secundarios y utilizada en cada una de las investigaciones se ha basado en la observación de Youtube España para captar datos a una hora determinada del día entre los vídeos más vistos. Para la consecución de cada objetivo secundario recurrimos a investigaciones con metodologías específicas que serán expuestas en el apartado de resultados de datos para facilitar la comprensión e interpretación de los Gráficos y Cuadros. El objetivo de este artículo puede ser discutible en las conclusiones finales que se obtengan ya que se rompen algunos convencionalismos. Sin embargo, también dejaremos abiertas algunas líneas de investigación que podrían analizarse en futuras publicaciones para rebatir algunas de nuestras conclusiones.

\section{Descripción cuantitativa del espectador de Youtube}

Antes de adentrarnos en los datos específicos de Youtube, observemos la información que existe sobre el internauta español. Internet tiene una penetración del $44,7 \%$ entre la población y un perfil de audiencia mucho más joven que el del resto de medios (AIMC, 2012b). En este sentido, el grupo de edad que más internautas acumula es el que comprende a personas entre 25 y 44 años (50,2\%). En el Gráfico 1 además observamos que son más hombres los internautas y que los usuarios destacan en las clases sociales más adineradas.

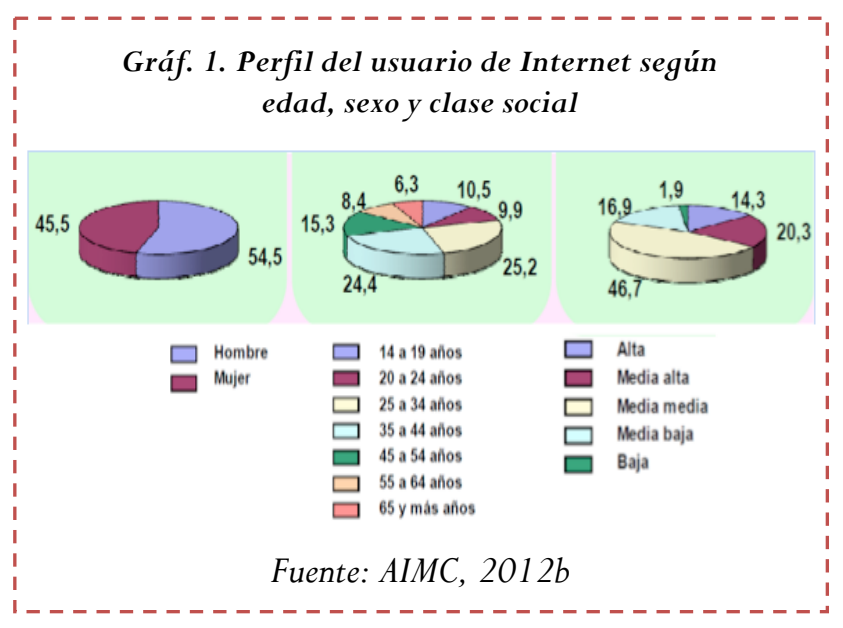

Sin embargo, existen diferencias notables entre el perfil del usuario de Internet con respecto al del medio televisivo como observamos en el Gráfico 2.

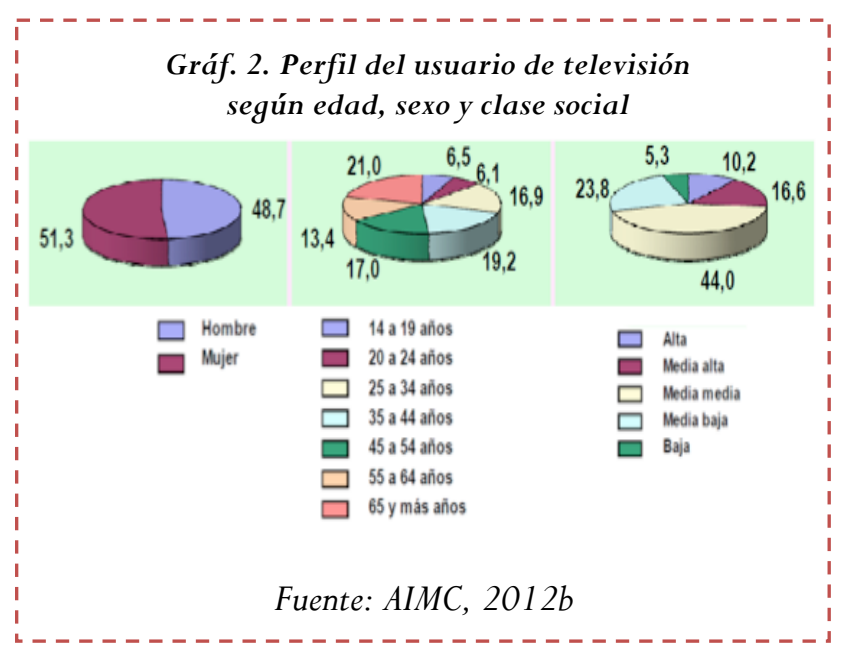

Por ejemplo, en la televisión los espectadores con 65 y más años suponen el $21 \%$ de los espectadores frente al 6,3\% en Internet. Además la presencia femenina es ligeramente superior a la masculina en la televisión y la clase media baja y baja tienen mayor presencia.

Pero centrándonos en datos específicos de Youtube. Alexa (2013), una de las empresas que mide las visitas de Internet, analiza el perfil de edad de los espectado- 
res de Youtube con respecto al usuario habitual de Internet. En el Gráfico 3 se analiza la presencia de los usuarios de Youtube por edad, género, número de hijos, nivel educativo y lugar de conexión: en verde se indica que los usuarios de Youtube están presentes en esa categoría por encima de la media de Internet y en rojo los que están por debajo.

\section{Gráf. 3. Análisis demográfico de Youtube.com}

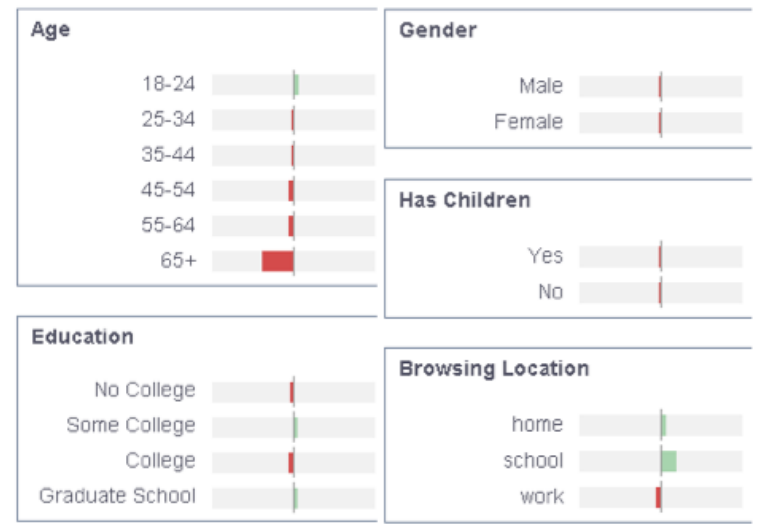

Fuente: Alexa, 2013

Observamos que la audiencia de Youtube entre 18 y 64 años es similar en porcentaje de consumo a la de Internet en su conjunto (Alexa, 2013). Sin embargo, la población mayor de 65 años está representada muy por debajo de la media de usuarios de la red. Es decir, si ya de por sí Internet es un medio demasiado joven y con escasa presencia del público adulto $(>65)$; Youtube se caracteriza por tener una audiencia más baja si cabe entre este grupo de población. En este sentido, es destacable que los usuarios de Youtube se conectan más desde casa y, principalmente, desde las instituciones educativas con respecto al resto de internautas. Algo que está directamente relacionado con la edad del usuario de esta red social.

Con respecto al sexo, los usuarios de Youtube están representados en el mismo porcentaje que en Internet; es decir, hay más hombres (59\%) que mujeres (Alexa, 2013, El Mundo, 2009 y AIMC, 2012b).

Pero Google (propietaria de Youtube) ofrece los datos de su audiencia según otro medidor de referencia: Nielsen. Sin embargo, limita estos resultados a su mercado local. Se tratan de datos de Estados Unidos ya que el departamento de comunicación de Youtube no ofrece estadísticas localizadas por países o regiones (se solicitaron para ésta y otras investigaciones sin éxito).

Youtube alcanza una audiencia de 128,2 millones de espectadores mensuales en Estados Unidos, de los que la mitad tiene entre 18 y 49 años (Nielsen, 2011). En este mismo estudio también queda patente el escaso peso del espectador con más de 65 años con apenas 10,9 millones de visitas mensuales. También es el grupo de edad con menos penetración entre los internautas con un $48 \%$. Sin embargo, Youtube destaca por situarse como la segunda red social con mayor penetración entre los usuarios de entre 18 y 55 años. Nielsen habla de marcas para clasificar a Youtube como la sexta web en la que los estadounidenses pasan más minutos al día (sólo superada por Facebook, Yahoo, Google, AOL Media Network y Bing).

En este mismo sentido, en el Gráfico 4 (IAB, 2011) observamos que tan solo Facebook arrasa en todas las franjas de edad con una clara primera posición en penetración entre los internautas.

Gráf. 4. Penetración de redes sociales en España según edad

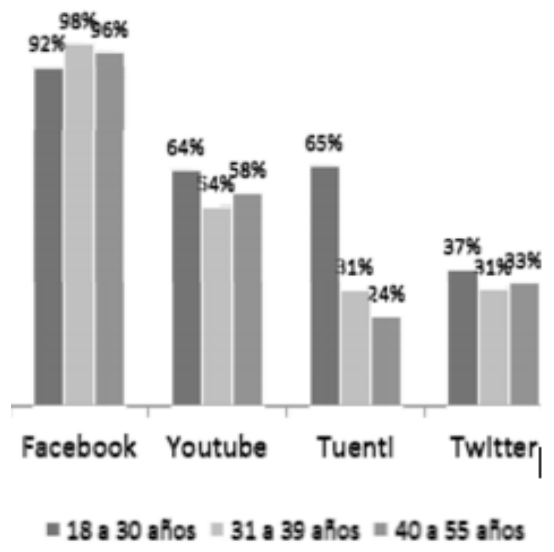

Fuente: $I A B, 2011$

Sin embargo, Youtube destaca con un $64 \%$ de penetración entre el público de entre 18 y 30 años. Tan solo superada por Tuenti en esa franja, una red social de contactos personales con un perfil muy juvenil y con penetración casi exclusiva en España. 


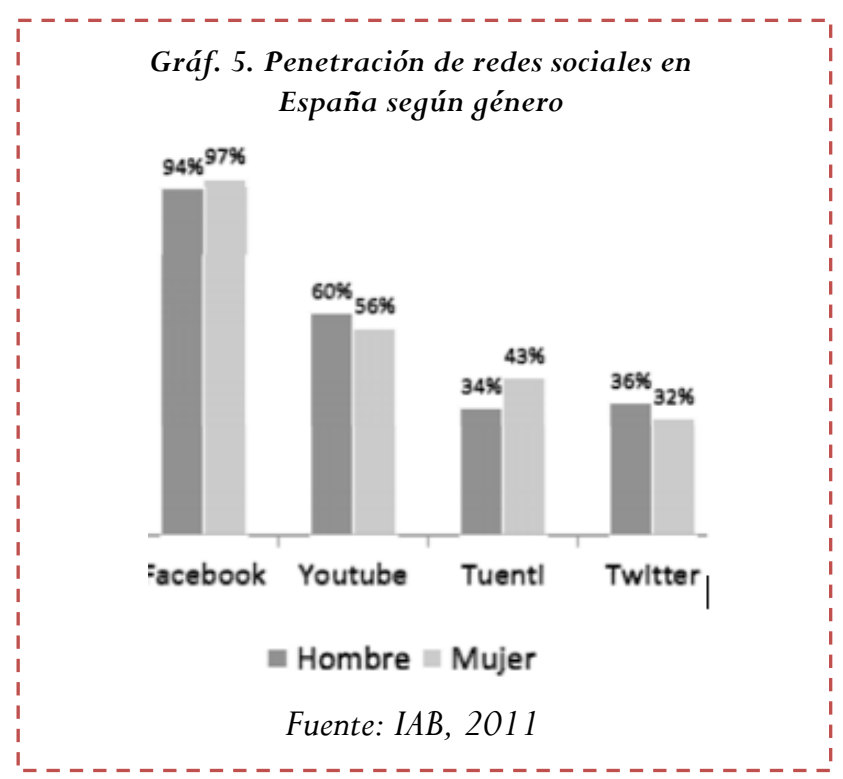

Con respecto al género, en el Gráfico 5 vemos que la penetración de esta red social es similar entre hombres y mujeres aunque, al igual que en el resto de estudios, ligeramente superior entre el género masculino.

\subsection{El perfil social del usuario de Youtube}

Las redes sociales se caracterizan por tener mayor penetración en el ámbito juvenil. Y, precisamente, la red social Youtube crece exponencialmente y cada año tiene más visitas por el arrastre de otras redes. Uno de sus motivos de crecimiento es el impulso que se le da desde las menciones de Twitter o Facebook (Aradas, 2012). Por ejemplo, según la propia web Youtube (2012) un tuit compartido genera seis nuevas sesiones de Youtube.com y cada minuto aparecen más de 500 tuits que enlazan con esta red de vídeos. De hecho, según Youtube (2012) cada día se reproducen en Facebook 500 años de vídeo de YouTube, y cada minuto se comparten en Twitter más de 700 vídeos.

En tanto en cuanto el uso de las redes sociales crece sin pausa se prevé un crecimiento proporcional de Youtube. De hecho, el uso de las redes sociales ha crecido en España un 24\% entre 2009 y 2011 según el informe del Interactive Advertising Bureau (IAB, 2011).

Esto podría justificar la escasa presencia del público más envejecido en Youtube ya que gran parte de su audiencia se genera desde redes sociales personales (también caracterizadas por tener un perfil más juvenil). El usuario prototipo de Youtube se conecta tres horas a la semana de media (IAB, 2011).

\section{Descripción cualitativa del espectador de Youtube}

Tras el apartado exclusivamente cuantitativo, pasamos a plasmar los datos relacionados con los tres objetivos secundarios para poder completar el objetivo principal de la investigación. Por tanto, a continuación expondremos los datos de la investigación que permiten describir el perfil cualitativo del espectador de vídeos en Youtube España.

\subsection{Resultados sobre el interés del espectador por lo glocal}

Este apartado se corresponde con el objetivo secundario (O1.1.) planteado en el artículo para demostrar que los usuarios de Youtube España prefieren los vídeos relacionados con su identidad cultural (local) a pesar de la potencialidad que le da este sitio global.
Recurriremos a los resultados del artículo "El caso Youtube España: el fenómeno glocal en las redes sociales" (Gallardo y Jorge, 2012). La investigación analiza una muestra de 405 vídeos recopilada a lo largo de 27 días consecutivos a las doce de la medianoche. En cada jornada se han analizado y recopilado los 15 vídeos más vistos, según la clasificación del propio sitio Youtube España, entre el 13 de noviembre de 2010 y el 9 de diciembre de 2010 (cuatro semanas).

Para ello se recopilaron tres rasgos en cada vídeo:

a) $\mathrm{Si}$ el texto de la descripción o del propio vídeo está en español.

b) Si el audio del vídeo está en español. 
c) Si el contenido del vídeo se refiere a un contenido vinculado con la identidad cultural española.

Los resultados fueron reveladores. En el Cuadro 1 observamos que el 77,8\% (315 vídeos) de los 405 vídeos de la muestra tiene alguno de los tres rasgos

\begin{tabular}{|c|c|}
\hline Videos & Videos \\
\hline extranjeros & españoles \\
\hline $315,77,8 \%$ & $90 ; 22,2 \%$ \\
\hline
\end{tabular}

Fuente: Gallardo y Jorge, 2012.

que lo identifican como español.

Sin embargo, en el Gráfico 6 es más destacable si cabe que el $69,7 \%$ de los vídeos analizados tenía todos los rasgos (tres de tres) para ser valorado español.

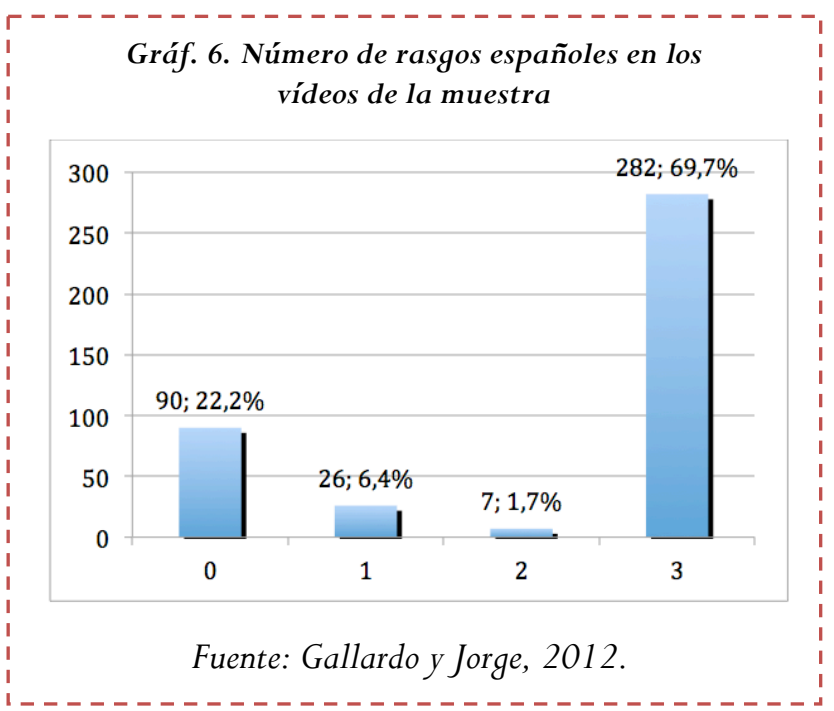

Es reseñable que en el trabajo de campo se han detectado referencias más localistas: de los 405 vídeos de la muestra se contabilizaron 15 vídeos con rasgos relacionados con comunidades autónomas de España: 14 de Cataluña y 1 de Galicia.

\subsection{Resultados sobre el interés del espectador por los contenidos televisivos}

Estos resultados se corresponden con el objetivo secundario O.1.2. relacionado con el interés del espectador de Youtube España por contenidos procedentes de la televisión. Nos centraremos en la investigación publicada en "La televisión tradicional quiere gobernar Internet: caso Youtube" (Gallardo, 2010) sobre una muestra de 465 vídeos analizados entre el 9 de enero y el 8 de febrero de 2008 (31 días); los datos se captaron diariamente entre los 15 vídeos más vistos a las doce de la noche. La publicación mide la influencia de las cadenas de televisión en Internet y para poder analizar el concepto de influencia se crea un índice de influencia de los contenidos de la televisión tradicional sobre el sitio web Youtube. Este índice de influencia es el resultado de la media de cuatro porcentajes obtenidos para cuantificar el peso de los contenidos procedentes de la televisión tradicional y que se consumen a través de Youtube:

a) Porcentaje de vídeos de la televisión en Youtube España de la muestra.

b) Porcentaje de visitas de vídeos de la televisión en Youtube España de la muestra.

c) Porcentaje de visitas de los 15 vídeos más vistos de cada muestra.

d) Porcentaje de horas de vídeos de la televisión en Youtube España de la muestra.

En esa investigación se captan dos muestras (diaria y semanal) para obtener de sus medias el índice conjunto de influencia de contenidos televisivos en Internet. La muestra mensual queda descartada porque en sus resultados interfieren más las visitas repetidas por los mismos usuarios (algo habitual en determinados tipos de contenidos: como los videoclips).

En el Cuadro 2 hemos hecho las medias de la muestra diaria y semanal (Gallardo, 2010) para poder observar de manera conjunta los datos en función del número de vídeos, del número de visitas de las 
muestras y de los 15 vídeos más vistos y del número de horas.

\begin{tabular}{|c|c|c|}
\hline \multicolumn{3}{|c|}{$\begin{array}{l}\text { Cuadro 2. Indice conjunto de influencia de } \\
\text { contenidos televisivos en Youtube España }\end{array}$} \\
\hline & $\begin{array}{l}\text { Vídeos de Tv en } \\
\text { Youtube }\end{array}$ & $\begin{array}{l}\text { Resto de vídeos } \\
\text { en Youtube }\end{array}$ \\
\hline $\begin{array}{l}\text { Número de } \\
\text { vídeos }\end{array}$ & $55 \%$ & $45 \%$ \\
\hline $\begin{array}{l}\text { Número de } \\
\text { visitas de toda la } \\
\text { muestra }\end{array}$ & $54,5 \%$ & $45,5 \%$ \\
\hline $\begin{array}{l}\text { Número de } \\
\text { visitas de } 15 \\
\text { vídeos más } \\
\text { vistos de las } \\
\text { muestras }\end{array}$ & $54,9 \%$ & $45,1 \%$ \\
\hline $\begin{array}{l}\text { Número de } \\
\text { horas }\end{array}$ & $70,5 \%$ & $29,5 \%$ \\
\hline $\begin{array}{l}\text { Media: índice del } \\
\text { peso de Tv en } \\
\text { Youtube }\end{array}$ & $58,7 \%$ & $41,3 \%$ \\
\hline \multicolumn{3}{|c|}{ Fuente: Elaboración propia a partir } \\
\hline de da & tos de Gallardo, & 010 \\
\hline
\end{tabular}

De esta manera, en el Cuadro 2 todos los vídeos procedentes de la televisión superan a los generados por los usuarios en los cuatro porcentajes analizados. La mayor diferencia se encuentra en función del número de horas (el 70,5\% del tiempo total de los vídeos analizados pertenece a vídeos de la televisión) lo que nos hace observar que los contenidos televisivos que se consumen por la red tienden a durar mucho más que el resto (el principal motivo es que los canales de las cadenas en Youtube reciben beneficios y ventajas para poder subir contenidos sin limitaciones).

De esta manera, el índice conjunto de influencia de la televisión en Youtube es de un 58,7\%. Dato relevante para valorar el peso de los contenidos televisivos en Internet. Por otra parte, si sacamos de esa muestra los contenidos de televisión procedentes de cadenas de fuera de España, el índice no baja sustancialmente: 53,5\%. Es decir, los contenidos televisivos de cadenas españolas tienen un peso relevante y prioritario en Youtube España. Lo que coincidiría con el punto anterior: lo local interesa más que lo global.

\subsection{Resultados sobre la pasividad del espec- tador}

A continuación expondremos los datos relacionados con la actitud del espectador de vídeos en Internet $y$, por ende, con el objetivo secundario O1.3. La primera barrera de interacción ante la que se encuentran los usuarios de Youtube es que es necesario estar registrado para poder interactuar. La investigación titulada "La baja interacción del espectador de vídeos en Internet: caso Youtube España” (Gallardo y Jorge, 2010) analiza cuatro muestras para cuantificar el grado de interactividad. Nosotros vemos suficiente, para describir al usuario, que nos centremos en dos muestras:

a) Muestra de los 15 vídeos más vistos del viernes 22 de junio de 2007 al viernes 28 de septiembre de 2007 (ambos incluidos). Se recopilaron los 15 vídeos más veces reproducidos los viernes a medianoche y sólo se registró el número de reproducciones y el de puntuaciones realizadas. Una muestra de 15 semanas y un total de 218 vídeos y 11.851 .877 visitas.

b) Muestra de los 15 vídeos más vistos desde el nacimiento de Youtube (2005) hasta febrero de 2008. Se recopilan los datos relacionados con el número de comentarios y puntuaciones dejados en cada vídeo. La muestra asciende a 15 vídeos, que acumulan 103.197.303 visitas.

En el Cuadro 3 (Gallardo y Jorge, 2010) observamos el promedio de visitas, de puntuaciones efectuadas y el promedio del porcentaje que suponen esas puntuaciones sobre el número de reproducciones.

Cuadro 3. Visitas, puntuaciones y peso de las puntuaciones (muestra de los 15 vídeos más vistos de junio a septiembre de 2007)

\begin{tabular}{|l|l|l|l|}
\hline & $N^{\circ}$ de visitas & $\begin{array}{l}\text { Puntuaciones } \\
\text { sobre vídeos } \\
\text { más vistos }\end{array}$ & $\begin{array}{l}\% \text { de puntuaciones } \\
\text { sobre } \\
\text { reproducciones de } \\
\text { vídeos }\end{array}$ \\
\hline Promedio & 54.366 & 91,5 & $0,18 \%$ \\
\hline
\end{tabular}

Fuente: Gallardo y Jorge, 2012. 
En el Cuadro 4 vemos que el porcentaje de personas que puntúa un vídeo es de tan sólo un $0,18 \%$ con una media de 91,5 puntuaciones cada 54.366 reproducciones.

\begin{tabular}{l|l|l|}
\hline \multicolumn{3}{c}{$\begin{array}{c}\text { Cuadro 4. Porcentaje de espectadores de } \\
\text { Youtube que dejan comentarios o puntúan } \\
\text { vídeos (muestra de los 15 vídeos más vistos } \\
\text { hasta febrero de 2008) }\end{array}$} \\
\hline
\end{tabular}

Observamos en este Cuadro 4 que sólo el 1,5 por mil de las personas que ve los vídeos decide dejar un comentario. Menos son aún las personas que valoran cada vídeo, algo que sólo hizo el 0,08\%.
El mismo estudio concluye que no se trata de una conducta que vaya a cambiar con el paso del tiempo. Es más, en un año de análisis la interacción adquiere una tendencia regresiva como vemos en el Gráfico 7.

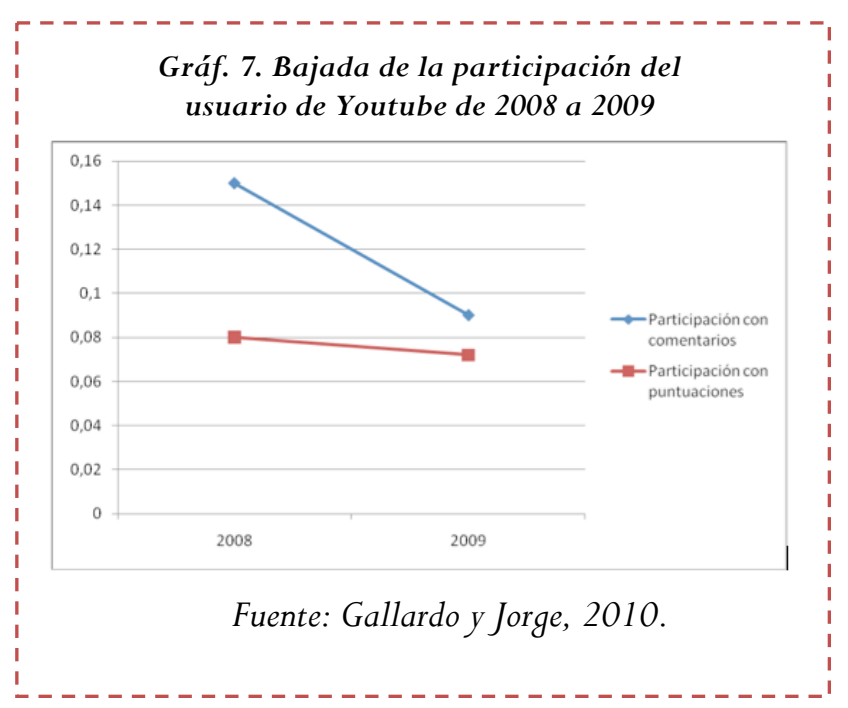

En cualquier caso estamos hablando de interacciones que están siempre por debajo de las dos décimas.

\section{Discusión de los resultados: el perfil del usuario de Youtube}

Con todos los datos expuestos hasta el momento es posible cumplir el objetivo principal: describir cuantitativa y cualitativamente el perfil del espectador de vídeos en Youtube España. Para ello ha sido relevante la información cuantitativa y el cumplimiento de los resultados referidos a los tres objetivos secundarios:

El O1.1. (referido al interés del espectador de Internet por lo glocal) se cumple ya que en el Cuadro 1 y Gráfico 6 vemos que los vídeos consumidos desde la ubicación España en Youtube están más relacionados con la identidad cultural local a pesar de que el internauta puede elegir entre un número en continuo crecimiento de vídeos procedentes de todo el mundo.

El O1.2. (referido al interés del espectador por los contenidos procedentes de la televisión) también se cumple a partir de la interpretación del índice conjunto de influencia de la televisión en Youtube: $58,7 \%$ (Cuadro 2). Hay una clara preponderancia de los contenidos procedentes de la televisión tradi- cional entre los vídeos más vistos, incluso, entre los procedentes de cadenas exclusivamente españolas. Lo que justifica más aún el interés por lo glocal del O1.1.

El O1.3. (referido a la escasa interacción del espectador de vídeos en Internet) también se cumple durante la visualización de contenidos subidos en Youtube. Hasta tal punto que la interacción no solo es baja sino que es casi inexistente con porcentajes de participación que no alcanzan ni siquiera el 1\% (Cuadros 3 y 4). Los usuarios no dejan comentarios, ni valoran y ni responden a los vídeos: prefieren ver. Sin embargo, este objetivo puede ponerse en duda ya que el comportamiento del mismo espectador podría cambiar cuando consume contenidos en directo. Es entonces donde sí adquiere un papel más activo y participativo a través de las redes sociales. Es decir, en función de la emisión del contenido (diferido o en directo) se motiva al espectador a dar sus opiniones ante la potencialidad no de interacción, sino de compartir sus vivencias ante una co- 
munidad que ve lo mismo que él. Aquí queda abierta una propuesta de nueva línea de investigación.

Ante estos resultados podemos dar por cumplido el objetivo principal del artículo. Describir el perfil del usuario tipo de Youtube:

- Hombre (Alexa, 2013; Nielsen, 2011; AIMC, 2012a, IAB, 2011).

- Entre 18 y 34 años (Alexa, 2013; Nielsen, 2011).

- O1.1. Interesado por los contenidos cercanos (Gallardo y Jorge, 2012) y usuario de smartphones $(38 \%)$ con capacidad de mayor localización del contenido (IAB, 2011).
- O1.1. Interesado por contenidos relacionados con su identidad cultural (Gallardo y Jorge, 2012).

- O1.2. Interesado por contenidos procedentes de las industrias culturales tradicionales: las cadenas de televisión, principalmente, de su país (Gallardo, 2010).

- O1.3. Carácter pasivo. Pocas ganas de interactuar cuando ve vídeos subidos en la red (Gallardo y Jorge, 2010). Aunque esta actitud podría variar durante el visionado de vídeos en directo.

\section{Conclusiones}

Ante esta descripción del usuario de Youtube, concluimos que el espectador traslada a Internet sus costumbres y formas de consumo. Es decir, no por tener un acceso potencial a la globalidad va a rechazar el interés por lo cercano. Y tampoco va a rechazar el placer de no interactuar y de limitarse a ver.

Tras la confirmación del perfil glocal del espectador, podemos concluir que en Internet se crean redes virtuales inspiradas en comunidades preexistentes y con limitaciones geográficas. Todo, con el fin de atraer usuarios que tienen en común una identidad cultural y que comparten vídeos vinculados a esa identidad común. Por tanto, las redes sociales están obligadas a localizar sus estrategias de expansión si quieren triunfar en cada uno de los mercados. Sobre todo, en un contexto en el que la penetración de los dispositivos móviles es cada vez mayor y crece la facilidad de localización de servicios y comunicaciones. Es decir, este cambio en las formas de consumo podría influir positivamente hacia la mayor interacción del usuario.

Sin embargo, hoy por hoy, al espectador de vídeos de Youtube le gusta los contenidos procedentes de las industrias culturales tradicionales. Las cadenas de televisión han trasladado su influencia a Internet. Nos encontramos ante un intento bien logrado de las empresas audiovisuales ya consolidadas y que ya dominan el mercado offline para conquistar la red.

Pero una de las conclusiones más polémicas y que abre la puerta para su ratificación en otras investiga- ciones es la referida al carácter pasivo del espectador de Youtube. La conclusión más inesperada es que existe una traslación de las formas de consumo de la televisión tradicional cuando el espectador ve vídeos por Internet y no interactúa (a pesar de las potencialidades que le ofrece la red). No obstante, la actitud del espectador cambia cuando ve contenidos en directo y es consciente de que su experiencia es compartida con otras personas. Es en este contexto, cuando el usuario puede adquirir un mayor papel de interacción. El fenómeno multipantalla (ver la televisión en la televisión mientras se usa el teléfono móvil o la tableta) está revolucionando este tipo de interacción.

En este sentido, Youtube está potenciando la emisión de contenidos en directo (por ejemplo, el discurso del Rey de España de 2012 se emitió en directo a través de su canal Casa Real TV en Youtube).

El espectador de televisión solo ha cambiado la pantalla en la que consume pero mantiene algunas de sus actitudes pasivas y sus gustos frente a la televisión tradicional. Como conclusión final, el consumo lineal de contenidos audiovisuales del proceso clásico de la comunicación (emisor-receptor) ha encontrado su continuidad, por el momento, en Internet (al menos en los contenidos grabados o subidos a la red). Algo que asegura la base de la industria audiovisual que solo ve peligrar su futuro por el modo de distribución y no de creación. 


\section{Referencias}

- $\operatorname{AIMC}(2012 \mathrm{a}):$ EGM: $2^{a}$ Ola 2012 Abril/Mayo. Disponible en: http://www.aimc.es/-Audienciade-Internet-en-el-EGM-.html (consultado el 20 de octubre de 2012).

- AIMC (2012b): Resumen general de resultados EGM, octubre de 2011 a mayo de 2012. Disponible en: http: / / www.aimc.es/-Datos-EGM-Resumen-

General-.html (consultado el 13 de octubre de 2012).

- Alexa (2013): Youtube.com’s Worldwide Rank, Disponible en: http: / / www.alexa.com/siteinfo/youtube.com (consultado el 02 de enero de 2013).

- Aradas, A. (2012): "Las redes sociales potencian el crecimiento de Youtube”. En: BBCMundo.com, tecnología, 24 de enero de 2012. Disponible en: http: / / www.bbc.co.uk/mundo/noticias/2012/0 1/120124_tecnologia_youtube_crecimiento_aa.sh tml (consultado el 10 de octubre de 2012).

- Burgess, J. E. y Green, J.B. (2009): Youtube: online video and participatory culture. Cambridge: Polity Press.

- Bustamante, E. (2006): Radio y Televisión en España: Historia de una asignatura pendiente de la democracia. Barcelona: GEDISA.

- Cebrián Herreros, M. (2004): Modelos de televisión: generalista, temática y convergente con Internet. Barcelona: Paidós.

- Díaz, R. (2009): "El vídeo en el ciberespacio: usos y lenguaje”. En: Comunicar: Revista científica iberoamericana de comunicación y educación, 33, Huelva, pp. 63-71.

- El Mundo (2009): Hombre maduro, de clase media y urbano, perfil del español que utiliza Youtube. En: Elmundo.es, 11/12/2009, Internet. Disponible en:

http: / /www.elmundo.es/elmundo/2009/12/11/ navegante/1260524364.html (consultado el 1 de noviembre de 2012).

- Fernández, S. (2002): "La glocalización de la comunicación”. En: Ámbitos, no 7-8, pp. 151-163. Disponible en: http: / / www.ull.es/publicaciones/latina/ambitos / 7-8/arti08.htm (consultado el 20 de noviembre de 2012).

- Gallardo, J. y Jorge, A. (2010): "La baja interacción del espectador de vídeos en Internet: caso Youtube España". En: Revista Latina de Comunicación Social, 65, pp. 421-435. Disponible en: http: / / www.revistalatinacs.org/10/art3/910_Ma laga/32_Gallardo.html (consultado el 21 de noviembre de 2012).

- Gallardo, J. (2010): La televisión tradicional quiere gobernar Internet. Caso Youtube. Madrid: Euroeditions.

- Gallardo, J. y Jorge, A. (2012): "El caso Youtube España: el fenómeno glocal en las redes sociales”. En: Revista Telos, $\mathrm{n}^{\circ}$ 92, Julio - Septiembre 2012. Disponible en: http: / / sociedadinformacion.fundacion.telefonica.c om/DYC/TELOS_92TELOS_EXPERIENCIAS/se ccion $=1271$ \&idioma $=$ es_ES\&id $=20120716120900$ 01\&activo $=6$.do (consultado el 20 de octubre de 2012).

- Geertz, C. (1988): La interpretación de las culturas. Barcelona: Gedisa.

- Horkheimer, M. y Adorno, T. (1994): Dialéctica de la ilustración: fragmentos filosóficos. Madrid: Trotta.

- IAB. (2010): II estudio sobre redes sociales en Internet. Noviembre de 2010, Interactive Advertising Bureau Spain Research. Disponible en: http: / / www.iabspain.net/ver.php?mod=descargas \&id_categoria $=4,14,40,17$ (consultado el 24 de noviembre de 2012).

- IAB. (2011): III estudio sobre redes sociales en Internet. Noviembre de 2011, Interactive Advertising Bureau Spain Research. Disponible en: http: / / www.iabspain.net/wpcontent/uploads/downloads/2012/04/IIIEstudio-sobre-redes-sociales-en-Internet.pdf (consultado 3 de enero de 2013).

- Kantar Media. (2013): Panel de Audiencia de TV. Disponible en: http://www.kantarmedia1.es/sections/product/p anel-audiencia (consultado el 02 de enero de 2013). 
- Lavado, A. (2010): "El consumo de Youtube en España”. En: Global Media Journal México, Volumen 7, Número 14, pp. 76-92. Disponible en: http: / / www.gmjei.com/journal/index.php/GMJ _EI/article/viewFile/308/260 (consultado el 20 de octubre de 2012).

- Mattelart, A. (1993): La comunicación-mundo. Historia de las ideas y de las estrategias. Madrid: Fundesco.

- Nielsen (2011): State of the media: The social media report, Q3, 2011. Disponible en: http: / / www.slideshare.net/tanmayms/nielsensocial-media-report-9250389 (consultado el 20 de octubre de 2012).

- Oliva, C. (2012): “Comunicación 2.0, visibilidad e interactividad: fundamentos de la imagen corporativa de las universidades públicas de Madrid en Youtube". En: Fonseca Journal of Communication, n ${ }^{\circ} 5$, pp $117 \quad-139$. Disponible en: http: / / fjc.usal.es/images/stories/fonseca/docume nts/articles/comunicacion_2_0.pdf (consultado el 2 de enero de 2012).

- O'Reilly, T. (2006): "Qué es web 2.0. Patrones del diseño y modelos del negocio para la siguiente generación del software”. En: Boletín de la Sociedad de la Información: Tecnología e Información, Tribuna, 23 de febrero de 2006, Disponible en: http: / / sociedadinformacion.fundacion.telefonica.c om/DYC/SHI/Articulos_Tribuna_Que_es_Web $\_20 /$ seccion $=1188$ \&idioma $=$ es_ES\&id $=20091001$ 16300061\&activo=4. do (consultado el 1 de noviembre de 2012).

- Owen, B. M. (2000): The Internet challenge to television. Massachusetts: Harvard.

- Pérez de Silva, J. (2000): La televisión ha muerto. La nueva producción audiovisual en la era de Internet: La tercera revolución industrial. Barcelona: GEDISA.

- Pérez, J. A. y Santos, T. (2009): "Las televisiones locales del País Vasco en Internet”. En: Revista Latina de Comunicación Social, 64, pp. 192-202. Disponible en: http: / /www.revistalatinacs.org/09/art/17_816_ 18_Bilbao/Perez_Dasilva_y_Santos_Diez.html (consultado el 12 de noviembre de 2012).

- Ritzer, G. (1996): Teoría Sociológica Contemporánea. New York: McGraw Hill.

- Robinson, J. P. y Geoffrey, G. (1997): Time for life: The surprising ways Americans use their time. Pennsylvania: Pennsylvania State Press.

- Tancer, B. (2008): Click, What Millions of People Do Online and Why It Matters. New York: Hyperion.

- Wolton, D. (2000): Internet, y después? Barcelona: Gedisa.

- Youtube (2012): Sala de prensa. Estadísticas 2012. Disponible en: http: / /www.youtube.com/t/press_statistics (consultado el 23 de octubre de 2012).

\section{Forma de Citación}

GALLARDO CAMACHO, Jorge: Descripción cuantitativa y cualitativa del espectador de videos en Youtube España. Revista Communication Papers, $\mathrm{N}^{\circ}$ 2, páginas 11 a 22. Departamento de Filología y Comunicación de la Universidad de Girona. Recuperado el __ de de 2 de: http://www.communicationpapers.es 